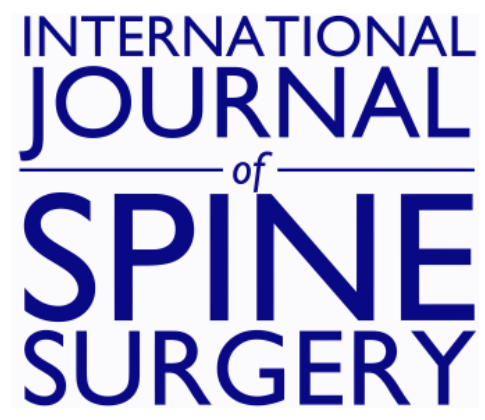

\title{
Increased Rates of Septic Shock, Cardiac Arrest, and Mortality Associated With Chronic Steroid Use Following Anterior Cervical Discectomy and Fusion for Cervical Stenosis
}

RYAN LEE, DANNY LEE, NIKHIL B. GOWDA, UCHECHI IWEALA, JEFFREY H.

WEINREB, DAVID P. FALK, WARREN YU and JOSEPH R. O'BRIEN

Int J Spine Surg 2020, 14 (5) 649-656

doi: https://doi.org/10.14444/7095

http://ijssurgery.com/content/14/5/649

This information is current as of April 26, 2023.

Email Alerts Receive free email-alerts when new articles cite this article. Sign up at: http://ijssurgery.com/alerts 


\title{
Increased Rates of Septic Shock, Cardiac Arrest, and Mortality Associated With Chronic Steroid Use Following Anterior Cervical Discectomy and Fusion for Cervical Stenosis
}

\author{
RYAN LEE, MBA, ${ }^{1}$ DANNY LEE, MD,${ }^{1}$ NIKHIL B. GOWDA, BS, ${ }^{1}$ UCHECHI IWEALA, MD, MBA, ${ }^{2}$ \\ JEFFREY H. WEINREB, MD, ${ }^{2}$ DAVID P. FALK, MD ${ }^{3}$ WARREN YU, MD, ${ }^{2}$ JOSEPH R. O'BRIEN, MD, \\ $\mathrm{MPH}^{4}$ \\ ${ }^{I}$ The George Washington University School of Medicine and Health Sciences, The George Washington University, Washington, District of Columbia, ${ }^{2}$ Department \\ of Orthopaedic Surgery, The George Washington University Hospital, Washington, District of Columbia, ${ }^{3}$ Department of Orthopaedic Surgery, University of \\ Pennsylvania Hospital, Philadelphia, Pennsylvania, ${ }^{4}$ Washington Spine and Scoliosis Clinic, OrthoBethesda, Bethesda, Maryland
}

\begin{abstract}
Objective: Anterior cervical discectomy and fusion (ACDF) is an established treatment modality for cervical spondylosis. Many patients are on immunosuppressant therapy in the management of various inflammatory spinal pathologies and other comorbid conditions. The impact of chronic steroid use on postoperative complications has not been examined in cervical fusion procedures. The objective of this study was to identify specific postoperative complications associated with steroid/immunosuppressant use following ACDF for cervical stenosis.

Methods: A multi-institutional surgical registry was queried to identify 5377 patients with ACDF diagnosed with cervical stenosis. Patients were stratified into cohorts with a history of steroid/immunosuppressant use for chronic conditions $(n=198,3.3 \%)$ versus those who did not $(n=5179,96.7 \%)$. Propensity-score matching without replacement was implemented to control for preoperative demographics and comorbidities. Pearson $\chi^{2}$ and Fischer exact tests were used in comparing the prevalence of demographics, comorbidities, and complication rates.

Results: Upon propensity matching, increased rates of pulmonary embolisms $(0.51 \% \mathrm{vs} 0.00 \%, P=.025)$, cardiac arrest requiring resuscitation $(1.01 \%$ vs $0.10 \%, P=.020)$, septic shock $(0.51 \%$ vs $0.00 \%, P=.025)$, and mortality $(1.52 \%$ vs $0.20 \%, P=.009)$ in the postoperative 30 -day period in patients on chronic steroid/immunosuppressant use were observed.

Conclusions: The results indicate that steroid use/immunosuppression in patients with ACDF has a higher associated rate of pulmonary embolisms, cardiac arrest, septic shock, and mortality. The risk of mortality and these other complications should be carefully considered prior to operative intervention. Future research may investigate steroid-tapering protocols that reduce the rate of infection and other postoperative complications in the subset of immunosuppressed ACDF patients.

Clinical Relevance: By elucidating the complication rates of ACDF patients on steroids for cervical stenosis, orthopedic surgeons can better stratify patients for risk of postoperative morbidity. Surgeons may have deeper riskbenefit discussions with these specific patients before they elect to have the operation.

Cervical Spine

Keywords: ACDF, cervical stenosis, steroids, complications
\end{abstract}

\section{INTRODUCTION}

Cervical spondylosis encompasses multiple degenerative changes of the cervical spine resulting in myelopathy and/or radiculopathy and has been classically associated with advancing age. ${ }^{1,2}$ With the incidence of cervical spondylosis and subsequent degenerative cervical myelopathy/radiculopathy expected to increase in the coming decades due to an aging population, the number of patients undergoing cervical spinal surgery is also expected to increase. ${ }^{3,4}$ For example, degenerative cervical myelopathy (DCM) is a strong indication for early operative intervention because patients can decline progressively in a predictable, stepwise fashion. Surgical management of DCM has also been shown to be cost-effective and to vastly improve patients' quality of life..$^{5}$

Many patients with degenerative cervical spine disease are on or have tried steroids to control 
inflammation and control symptoms. ${ }^{6}$ Although anterior cervical discectomy and fusion (ACDF) has been validated as an effective surgical modality for treating degenerative cervical spine disease with favorable postoperative outcomes, ${ }^{7}$ complication rates associated with the ACDF approach have not been studied in immunosuppressed patient populations. Many immunosuppressants, such as steroids and calcineurin inhibitors, have been linked with decreases in bone mineral density, ${ }^{8}$ potentially increasing risks of complications or failure after a cervical procedure. This study will analyze the effects of chronic steroid/immunosuppressant use for chronic conditions on postoperative complications in ACDF for the treatment of cervical spondylosis resulting in cervical spinal stenosis. Because surgeons use arthrodesis to treat a significant portion of patients with inflammatory degenerative cervical diseases, it is important to understand how steroid/immunosuppressant use affects complication and success rates in ACDF in this specific population so that surgeons can take proper preoperative and postoperative measures to optimize outcomes and patient satisfaction. We hypothesize that chronic steroid/immunosuppression therapy will predispose patients to various postoperative complications, particularly infectious complications.

\section{MATERIALS AND METHODS}

\section{Patient Cohort Selection}

The American College of Surgeons (ACS) maintains a large, multi-institutional clinical registry of all patients undergoing surgical procedures at one of 680 participating institutions in its National Surgical Quality Improvement Program (NSQIP) database. We queried the NSQIP database to identify and isolate all patients who had undergone an ACDF procedure, using Current Procedure Terminology (CPT) codes corresponding with 22551 and 22554. A total of 38645 patients who had undergone ACDF from 2005 to 2016 were isolated for this retrospective analysis. Patients were then selected if they had a diagnosis of cervical stenosis, which was identified by International Classification of Diseases $(I C D)$ codes. Patients who had a postoperative diagnosis $I C D-9$ and/or $I C D$ - 10 code corresponding with 723 and M48.02, respectively, were included for analysis. Patients excluded from the study were those with incomplete demographic information (2758) and those who also underwent posterior cervical fusion, laminectomies, facetectomies, and foraminotomies of the cervical vertebral bodies. After all inclusion and exclusion criteria were applied, 5377 patients with ACDF who were diagnosed with cervical stenosis were ultimately included in the current study. These patients were then stratified into 2 separate cohorts - those who had a history of steroid/immunosuppressant use for chronic conditions $(\mathrm{n}=198,3.3 \%)$ versus those who did not $(\mathrm{n}=5179,96.7 \%)$. Steroid use is defined by the ACS NSQIP database as "requiring regular administration of oral or parenteral corticosteroid (e.g. prednisone, Decadron) medications or immunosuppressant medications, within the 30 days prior to the principal operative procedure or at the time the patient is being considered as a candidate for surgery, for a chronic medical condition (e.g. COPD [chronic obstructive pulmonary disease], asthma, rheumatologic disease, rheumatoid arthritis, inflammatory bowel disease). A one-time pulse, limited short course, or a taper of less than 10 days duration would not qualify. The variable does not include topical corticosteroids applied to the skin or corticosteroids administered by inhalation or rectally, nor does it include short course steroids (duration 10 days or less) in the 30 days prior to surgery." 9

\section{Variables}

Demographic factors and preoperative comorbidities were included in this study to gain population-based insights into the differences associated with steroid use before undergoing ACDF. Demographic factors include age, sex, race, and body mass index $\left(\mathrm{kg} / \mathrm{m}^{2} ; \mathrm{BMI}\right)$. Preoperative comorbidities include diabetes mellitus, smoking history, dyspnea, COPD, congestive heart failure, hypertension, acute renal failure, dialysis dependence, disseminated cancer, open wound or wound infections, weight loss of more than $10 \%$ within 6 months prior to surgery, hematologic disorders (eg, vitamin K deficiency, thrombocytopenia), systemic sepsis, and functional dependence. ${ }^{9}$ Also included were perioperative variables, such as time of surgical delay from admission to operation, operative time, total length of hospital stay, and American Society of Anesthesiologists (ASA) class.

Postoperative adverse events analyzed in this study include the occurrence of surgical site infections, wound dehiscence, pneumonia, un- 
planned intubation, pulmonary embolism (PE), ventilator dependence ( $\geq 48$ hours), progressive renal insufficiency, acute renal failure, urinary tract infections, strokes, cardiac arresting requiring cardiopulmonary resuscitation, myocardial infarctions, blood transfusions, deep venous thromboembolisms (DVT), systemic sepsis, septic shock, return to the operating room, extended length of stay (LOS; $\geq 5$ days), unplanned readmission, and mortality within the 30-day period following ACDF.

\section{Statistical Analysis}

To determine differences in patient demographic factors, comorbidities, and perioperative/postoperative outcomes, univariate analyses were first used to assess statistical difference in prevalence of specific variables, stratified into 2 cohorts of patients - those with and those without a history of chronic steroid use. Pearson $\chi^{2}$ tests were used to test for differences in categorical variables, whereas 1-way analysis of variance was used to assess differences in mean values of continuous variables such as age, laboratory values, and time. Whereas these continuous variables were expressed as mean values with respective standard deviations, categorical variables were reported as incidence rates within the respective cohorts.

Propensity-score matching without replacement was implemented in a 1:5 manner, controlling for the significantly different patient demographic factors and preoperative comorbidities, to generate a control cohort of patients who were not taking steroids for more accurate comparison with those ACDF patients with cervical stenosis who were using steroids within the 30 days leading up to the procedure. Due to the smaller size of the steroid-use cohort ( $\mathrm{n}=198$ patients with a history of steroid use), matching was conducted in a 1:5 ratio to generate a propensity-matched control cohort of 990 patients to minimize the chances of high-quality control matches being discarded due to the small number of steroid-use patients. The caliber was set to 0.2 , with all variables that were being controlled for being entered as "covariates," when matching. Propensity-matching was performed using R, version 3.3.3.

All statistical findings with $P$ values less than or equal to .05 were considered significant in this analysis. All statistical analyses were performed using the SPSS Statistics, version 25, software (IBM
Corporation, Armonk, NY) and R Version 3.3.3 (The R Foundation, Auckland, New Zealand).

\section{RESULTS}

Among the 38645 ACDF patients analyzed, $5377(13.91 \%)$ patients had a postoperative diagnosis of cervical stenosis; of these, 198 (3.68\%) had a history of steroid use in the management of a chronic condition in the 30-day preoperative period. In the initial univariate analyses, the steroid cohort was significantly older (59.78 years vs 56.09 years, $P$ $<.001$ ) and had a larger proportion of female patients $(58.59 \%$ vs $49.53 \%, P=.012)$ than the control cohort (Table 1). Patients using steroids were more likely than those without a history of steroid use to present with dyspnea $(10.61 \%$ vs $5.41 \%, P=.007)$, COPD $(8.08 \%$ vs $4.69 \%, P=$ $.029)$, hypertension requiring medication management $(59.60 \%$ vs $46.89 \%, P=.001)$, acute renal failure $(0.51 \%$ vs $0.00 \%, P<.001)$, dialysis dependence $(1.01 \%$ vs $0.23 \%, P=.035)$, disseminated cancer $(1.01 \%$ vs $0.08 \%, P<.001)$, and an open wound or wound infection preoperatively $(1.01 \%$ vs $0.23 \%, P=.035)$ in the unmatched analysis (Table 1).

In the propensity-matched analysis, the steroid cohort had significantly higher rates of dyspnea $(10.61 \%$ vs $6.16 \%, P=.032)$, acute renal failure $(0.51 \%$ vs $0.00 \%, P=.025)$, and disseminated cancer preoperatively $(1.01 \%$ vs $0.10 \%, P=.020)$, with no differences in age, gender, race/ethnicity, or BMI (Table 1).

Patients with a history of steroid use experienced a significantly longer length of total hospital stay (1.61 days vs 2.16 days, $P=.007$ ) and a significantly longer delay from admission to operation ( 0.05 days vs 0.44 days, $P=.009$ ) when compared with the propensity-generated control cohort. A larger proportion of the steroid cohort $(61.62 \%)$ was assigned an ASA class of 3 or higher than was the propensitymatched control cohort $(50.00 \%, P<.001$; Table 2).

Those on steroids had higher postoperative complication rates when compared with the propensity-adjusted patients, including PEs $(0.51 \%$ vs $0.00 \%, P=.025)$, cardiac arrest requiring resuscitation $(1.01 \%$ vs $0.10 \%, P=.020)$, septic shock $(0.51 \%$ vs $0.00 \%, P=.025)$, and mortality $(1.52 \%$ vs $0.20 \%, P=.009)$ in the 30 -day period following ACDF (Table 3). Similar trends were seen in complication rates when comparing steroid users 
Table 1. Demographics and preoperative comorbidities in ACDF patients with cervical stenosis.

\begin{tabular}{|c|c|c|c|c|c|c|c|c|}
\hline \multirow[b]{2}{*}{ Demographics } & \multicolumn{2}{|c|}{ Control $(n=5179)$} & \multicolumn{2}{|c|}{ PM Control $(\mathbf{n}=990)^{*}$} & \multicolumn{2}{|c|}{ Steroid Use $(n=198)$} & \multirow[t]{2}{*}{$P$ Value } & \multirow[t]{2}{*}{ PM $P$ Value } \\
\hline & \multirow{2}{*}{\multicolumn{2}{|c|}{$56.09 \pm 11.048$}} & \multirow{2}{*}{\multicolumn{2}{|c|}{$59.84 \pm 10.178$}} & \multirow{2}{*}{\multicolumn{2}{|c|}{$59.78 \pm 10.967$}} & & \\
\hline Age, $y^{\dagger}$ & & & & & & & \multirow{4}{*}{$\begin{array}{r}<.001 \\
.012\end{array}$} & \multirow{4}{*}{$\begin{array}{l}.948 \\
.895\end{array}$} \\
\hline Sex & & & & & & & & \\
\hline Female & 2565 & $(49.53)$ & 575 & $(58.08)$ & 116 & $(58.59$ & & \\
\hline Male & 2614 & $(50.47)$ & 415 & $(41.92)$ & 82 & (41.41) & & \\
\hline Race & & & & & & & .270 & .853 \\
\hline American Indian or Alaska Native & 53 & $(1.02)$ & 8 & $(0.81)$ & 1 & $(0.51)$ & & \\
\hline Asian or Pacific Islander & 103 & (1.99) & 8 & $(0.81)$ & 1 & $(0.51)$ & & \\
\hline Black or African American & 501 & $(9.67)$ & 101 & $(10.20)$ & 22 & (11.11) & & \\
\hline Hispanic & 1 & $(0.02$ & 24 & $(2.42)$ & 7 & $(3.54)$ & & \\
\hline White & 4173 & $(80.58$ & 849 & $(85.76)$ & 167 & $(84.34)$ & & \\
\hline Body mass index $\left(\mathrm{kg} / \mathrm{m}^{2}\right)$ & & & & & & & .818 & .913 \\
\hline Normal $(<25.0)$ & 1054 & $(20.35$ & 176 & $(17.78)$ & 34 & $(17.17)$ & & \\
\hline Overweight (25.0-29.9) & 1704 & $(32.90$ & 320 & $(32.32)$ & 70 & $(35.35)$ & & \\
\hline Class I $(30.0-34.9)$ & 1344 & $(25.95$ & 254 & $(25.66)$ & 50 & $(25.25)$ & & \\
\hline Class II $(35.0-39.9)$ & 649 & $(12.53)$ & 133 & $(13.43)$ & 26 & $(13.13)$ & & \\
\hline Class III $(>40.0)$ & 428 & $(8.26)$ & 107 & $(10.81)$ & 18 & $(9.09)$ & & \\
\hline Preoperative comorbidities & & & & & & & & \\
\hline Diabetes mellitus & & & & & & & .453 & .148 \\
\hline No diabetes mellitus & 4328 & $(83.57)$ & 794 & $(80.20)$ & 170 & $(85.86)$ & & \\
\hline Non-insulin dependent & 563 & $(10.87)$ & 125 & (12.63) & 16 & $(8.08)$ & & \\
\hline Insulin dependent & 288 & $(5.56)$ & 71 & $(7.17)$ & 12 & $(6.06)$ & & \\
\hline Smoke & 1414 & $(27.30)$ & 236 & $(23.84)$ & 40 & $(20.20)$ & .027 & .269 \\
\hline Dyspnea & & & & & & & .007 & .032 \\
\hline No dyspnea & 4899 & (94.59) & 929 & $(93.84$ & 177 & (89.39) & & \\
\hline Moderate exertion & 259 & $(5.00)$ & 59 & $(5.96$ & 19 & $(9.60)$ & & \\
\hline At rest & 21 & $(0.41)$ & 2 & $(0.20)$ & 2 & $(1.01)$ & & \\
\hline COPD & 243 & $(4.69)$ & 58 & $(5.86)$ & 16 & $(8.08)$ & .029 & .238 \\
\hline Congestive heart failure & 5 & $(0.10)$ & 2 & $(0.20)$ & 0 & $(0.00)$ & .662 & .527 \\
\hline Hypertension & 2480 & $(47.89)$ & 543 & $(54.85)$ & 118 & $(59.60)$ & .001 & 0.220 \\
\hline Acute renal failure & 0 & $(0.00)$ & 0 & $(0.00)$ & 1 & $(0.51)$ & $<.001$ & 0.025 \\
\hline Dialysis & 12 & $(0.23)$ & 4 & $(0.40)$ & 2 & $(1.01)$ & .035 & 0.272 \\
\hline Disseminated cancer & 4 & $(0.08)$ & 1 & $(0.10)$ & 2 & $(1.01)$ & $<.001$ & .020 \\
\hline Wound infection & 12 & $(0.23)$ & 2 & $(0.20)$ & 2 & $(1.01)$ & .035 & .073 \\
\hline Hematologic disorders & 56 & $(1.08)$ & 13 & $(1.31)$ & 5 & $(2.53)$ & .060 & .202 \\
\hline Systemic sepsis & 5 & $(0.10)$ & 0 & $(0.00)$ & 0 & $(0.00)$ & .662 & $\ldots$ \\
\hline Functional status & & & & & & & .328 & .287 \\
\hline Independent & 5098 & (98.44) & 979 & $(98.89)$ & 193 & (97.47) & & \\
\hline Partially dependent & 74 & $(1.43)$ & 9 & $(0.91)$ & 4 & $(2.02)$ & & \\
\hline Totally dependent & 7 & $(0.14)$ & 2 & $(0.20)$ & 1 & $(0.51)$ & & \\
\hline
\end{tabular}

Abbreviations: ASA, American Society of Anesthesiologists; COPD, chronic obstructive pulmonary disease; PM, propensity- matched.

*PM control cohort was generated by controlling for age, sex, smoking, COPD, dyspnea, hypertension, acute renal failure, disseminated cancer, wound infections, dialysis, ASA.

$\dagger$ Values for age expressed as mean \pm standard deviation; all other values expressed as $\mathrm{n}(\%)$.

Bold values indicate statistical significance at $\mathrm{p}<0.05$.

in the propensity-matched cohorts, stratified by 1and 2-level ACDF versus 3-level or higher ACDF. Similar complication rates were observed in the 1and 2-level ACDF cohort, specifically (Table 4).

\section{DISCUSSION}

In the United States, a move towards bundled payments/risk sharing makes understanding the factors that affect rates of complications and LOS crucial. This study has analyzed chronic presurgical steroid use and associated comorbidities. The results of this study demonstrate that steroid use for chronic medical conditions is associated with a number of preoperative morbidities and also with higher rates of multiple postoperative complications. Consideration of these results may make the surgeon adverse to surgical intervention, but not all surgical management for cervical spinal stenosis is elective. As such, these data should be used to inform those creating bundled payment plans with hospitals and insurers.

The present study demonstrated longer LOS for those on preoperative chronic steroid therapy undergoing $\mathrm{ACDF}$ for cervical stenosis than their propensity-matched cohorts. These findings are in line with the current literature, given that White et $\mathrm{al}^{10}$ previously reported similar increases in LOS for patients on preoperative chronic steroids for anterior lumbar fusion. It is likely that patients on steroid therapy are medically more complex and may understandably require resources at the time of surgery. For example, chronic steroid use suppresses 
Table 2. Comparison of perioperative variables stratified by steroid use.

\begin{tabular}{|c|c|c|c|c|c|}
\hline Operative Variables & Control $(n=5179)$ & PM Control Cohort $(\mathbf{n}=990) *$ & Steroid Use $(n=198)$ & $P$ Value & PM $P$ Value* \\
\hline Days to operation from admission $\uparrow$ & $0.04 \pm 0.552$ & $0.05 \pm 0.738$ & $0.44 \pm 4.399$ & $<.001$ & .009 \\
\hline Total operating time (minutes) $\dagger$ & $132.99 \pm 70.918$ & $131.67 \pm 67.632$ & $134.03 \pm 68.506$ & .839 & .654 \\
\hline Total hospital stay length (days) $\dagger$ & $1.66 \pm 2.392$ & $1.61 \pm 1.825$ & $2.16 \pm 5.006$ & .006 & .007 \\
\hline Care type, $\mathrm{n}(\%)$ & & & & .702 & .871 \\
\hline Inpatient & $4074(78.66)$ & $795(80.30)$ & $158(79.80)$ & & \\
\hline Outpatient & $1105(21.34)$ & $195(19.70)$ & $40(20.20)$ & & \\
\hline Anesthesia administered & & & & .750 & .655 \\
\hline General & $5164(99.71)$ & $989(99.90)$ & $198(100.00)$ & & \\
\hline Spinal & $7(0.14)$ & $0(0.00)$ & $0(0.00)$ & & \\
\hline Other & $8(0.15)$ & $1(0.10)$ & $0(0.00)$ & & \\
\hline ASA classification & & & & $<.001$ & $<.001$ \\
\hline 1-No disturb & $144(2.78)$ & $24(2.42)$ & $0(0.00)$ & & \\
\hline 2-Mild disturb & $2781(53.70)$ & $471(47.58)$ & $76(38.38)$ & & \\
\hline 3-Severe disturb & $2177(42.04)$ & $481(48.59)$ & $110(55.56)$ & & \\
\hline 4-Life threat & $73(1.41)$ & $13(1.31)$ & $12(6.06)$ & & \\
\hline 5-Moribund & $4(0.08)$ & $1(0.10)$ & $0(0.00)$ & & \\
\hline
\end{tabular}

Abbreviation: ASA, American Society of Anesthesiologists; PM, propensity-matched.

*PM control cohort was generated by controlling for age, sex, smoking, COPD, dyspnea, hypertension, acute renal failure, disseminated cancer, wound infections, dialysis, ASA.

$\dagger$ Values expressed as mean \pm standard deviation; all other values expressed as $n(\%)$.

Bold values indicate statistical significance at $\mathrm{p}<0.05$.

the hypothalamic-pituitary-adrenal axis and can lead to subsequent adrenal insufficiency. Glucocorticoid stress dosing to prevent adrenal crisis may be one of many such considerations regarding chronic steroid therapy that may be responsible for increasing the duration from admission to operation for these medically complex patients. However, there is a lack of consensus and ongoing debate regarding the use of perioperative stress dosing. ${ }^{11,12}$
In line with the current literature, this study demonstrated increased rates of PE in patients on steroid/immunosuppressive therapy undergoing ACDF for cervical stenosis. Previous analyses of nationwide databases and pharmacological registries have demonstrated increased rates of PE in patients on chronic steroid therapy in the general population. ${ }^{13,14}$ Chronic steroid therapy has also demonstrated increased PE risk in the 30-day postoperative period for neurosurgical proce-

Table 3. Propensity-matched univariate analyses for postoperative complications by steroid use.

\begin{tabular}{|c|c|c|c|c|c|}
\hline Postoperative Complications & Control $(n=5179)$ & PM Control $(\mathbf{n}=990)^{*}$ & Steroid Use $(n=198)$ & $P$ Value & PM $P$ Value* \\
\hline \multicolumn{6}{|l|}{ Surgical site infections } \\
\hline Superficial incisional & $15(0.29)$ & $3(0.30)$ & $1(0.51)$ & 0.585 & 0.654 \\
\hline Deep incisional & $13(0.25)$ & $6(0.61)$ & $0(0.00)$ & 0.480 & 0.272 \\
\hline Organ/space & $5(0.10)$ & $2(0.20)$ & $1(0.51)$ & 0.091 & 0.438 \\
\hline Wound disruption & $2(0.04)$ & $0(0.00)$ & $0(0.00)$ & 0.782 & $\ldots$ \\
\hline Pneumonia & $35(0.68)$ & $9(0.91)$ & $3(1.52)$ & 0.166 & 0.436 \\
\hline Unplanned intubation & $27(0.52)$ & $5(0.51)$ & $1(0.51)$ & 0.975 & 1.000 \\
\hline Pulmonary embolism & $8(0.15)$ & $0(0.00)$ & $1(0.51)$ & .236 & .025 \\
\hline Ventilator dependence $(>48 \mathrm{~h})$ & $7(0.14)$ & $0(0.00)$ & $0(0.00)$ & .605 & $\ldots$ \\
\hline Progressive renal insufficiency & $2(0.04)$ & $0(0.00)$ & $0(0.00)$ & .782 & $\ldots$ \\
\hline Acute renal failure & $1(0.02)$ & $0(0.00)$ & $0(0.00)$ & .845 & $\ldots$ \\
\hline Urinary tract infection & $23(0.44)$ & $7(0.71)$ & $1(0.51)$ & .900 & .751 \\
\hline CVA/Stroke & $5(0.10)$ & $1(0.10)$ & $1(0.51)$ & .091 & .206 \\
\hline Cardiac arrest & $6(0.12)$ & $1(0.10)$ & $2(1.01)$ & .001 & .020 \\
\hline Myocardial infarction & $9(0.17)$ & $3(0.30)$ & $0(0.00)$ & .557 & .438 \\
\hline Blood transfusions & $18(0.35)$ & $4(0.40)$ & $0(0.00)$ & .406 & .370 \\
\hline Deep venous thromboembolism & $16(0.31)$ & $3(0.30)$ & $1(0.51)$ & .630 & .654 \\
\hline \multicolumn{6}{|l|}{ Septic complications } \\
\hline Systemic sepsis & $11(0.21)$ & $3(0.30)$ & $1(0.51)$ & .392 & .654 \\
\hline Septic shock & $3(0.06)$ & $0(0.00)$ & $1(0.51)$ & .024 & .025 \\
\hline Reoperation & $81(1.56)$ & $12(1.21)$ & $3(1.52)$ & .957 & .727 \\
\hline Extended length of stay $(\geq 5 \mathrm{~d})$ & $207(4.00)$ & $30(3.03)$ & $7(3.54)$ & .744 & .709 \\
\hline Unplanned readmission & $177(3.42)$ & $35(3.54)$ & $8(4.04)$ & .637 & .728 \\
\hline Death & $10(0.19)$ & $2(0.20)$ & $3(1.52)$ & $<.001$ & .009 \\
\hline
\end{tabular}

Abbreviations: ASA, American Anesthesiologists Association; COPD, chronic obstructive pulmonary disease; CVA, cerebral vascular accident; PM, propensity-matched. *PM control cohort was generated by controlling for age, sex, smoking, COPD, dyspnea, hypertension, acute renal failure, disseminated cancer, wound infections, dialysis, ASA.

Bold values indicate statistical significance at $\mathrm{p}<0.05$. 
Table 4. Propensity-matched univariate analyses for postoperative complications stratified by number of levels fused.

\begin{tabular}{|c|c|c|c|c|c|c|}
\hline \multirow[b]{2}{*}{ Postoperative Complications } & \multicolumn{3}{|c|}{1 and 2 Levels Fused } & \multicolumn{3}{|c|}{3 or More Levels Fused } \\
\hline & $\begin{array}{c}\text { PM Control, } \\
\text { n }(\%), \mathbf{n}=904 \%\end{array}$ & $\begin{array}{c}\text { Steroid Use, } \\
\mathrm{n}(\%), \mathrm{n}=185\end{array}$ & $P$ Value & $\begin{array}{l}\text { PM Control, } \\
\mathbf{n}(\%), \mathbf{n}=\mathbf{8 6} \%\end{array}$ & $\begin{array}{c}\text { Steroid Use, } \\
\mathrm{n}(\%), \mathrm{n}=13\end{array}$ & $P$ Value \\
\hline \multicolumn{7}{|l|}{ Surgical site infections } \\
\hline Superficial incisional & $3(0.3)$ & $1(0.5)$ & .526 & $0(0.0)$ & $0(0.0)$ & $\ldots$ \\
\hline Deep incisional & $4(0.4)$ & $0(0.0)$ & 1.000 & $2(2.3)$ & $0(0.0)$ & 1.000 \\
\hline Organ/space & $2(0.2)$ & $1(0.5)$ & .428 & $0(0.0)$ & $0(0.0)$ & $\ldots$ \\
\hline Wound disruption & $0(0.0)$ & $0(0.0)$ & $\ldots$ & $0(0.0)$ & $0(0.0)$ & $\ldots$ \\
\hline Pneumonia & $8(0.9)$ & $2(1.1)$ & .682 & $1(1.2)$ & $1(7.7)$ & .247 \\
\hline Unplanned intubation & $5(0.6)$ & $1(0.5)$ & .983 & $0(0.0)$ & $0(0.0)$ & $\ldots$ \\
\hline Pulmonary embolism & $0(0.0)$ & $1(0.5)$ & .027 & $0(0.0)$ & $0(0.0)$ & $\ldots$ \\
\hline Ventilator dependence $(>48 \mathrm{~h})$ & $0(0.0)$ & $0(0.0)$ & $\ldots$ & $0(0.0)$ & $0(0.0)$ & $\ldots$ \\
\hline Progressive renal insufficiency & $0(0.0)$ & $0(0.0)$ & $\ldots$ & $0(0.0)$ & $0(0.0)$ & $\ldots$ \\
\hline Acute renal failure & $0(0.0)$ & $0(0.0)$ & $\ldots$ & $0(0.0)$ & $0(0.0)$ & $\ldots$ \\
\hline Urinary tract infection & $6(0.7)$ & $1(0.5)$ & .849 & $1(1.2)$ & $0(0.0)$ & 1.000 \\
\hline $\mathrm{CVA} /$ stroke & $1(0.1)$ & $1(0.5)$ & .213 & $0(0.0)$ & $0(0.0)$ & $\ldots$ \\
\hline Cardiac arrest & $1(0.1)$ & $2(1.1)$ & .022 & $0(0.0)$ & $0(0.0)$ & $\ldots$ \\
\hline Myocardial infarction & $3(0.3)$ & $0(0.0)$ & 1.000 & $0(0.0)$ & $0(0.0)$ & $\ldots$ \\
\hline Blood transfusions & $3(0.3)$ & $0(0.0)$ & 1.000 & $1(1.2)$ & $0(0.0)$ & 1.000 \\
\hline Deep venous thromboembolism & $2(0.2)$ & $1(0.5)$ & .428 & $1(1.2)$ & $0(0.0)$ & 1.000 \\
\hline \multicolumn{7}{|l|}{ Septic complications } \\
\hline Systemic sepsis & $2(0.2)$ & $1(0.5)$ & .428 & $1(1.2)$ & $0(0.0)$ & 1.000 \\
\hline Septic shock & $0(0.0)$ & $1(0.5)$ & .027 & $0(0.0)$ & $0(0.0)$ & $\ldots$ \\
\hline Reoperation & $9(1.0)$ & $3(1.6)$ & .440 & $3(3.5)$ & $0(0.0)$ & 1.000 \\
\hline Extended length of stay $(\geq 5 \mathrm{~d})$ & $27(3.0)$ & $7(3.8)$ & .570 & $3(3.5)$ & $0(0.0)$ & 1.000 \\
\hline Unplanned readmission & $31(3.4)$ & $8(4.3)$ & .551 & $4(4.7)$ & $0(0.0)$ & 1.000 \\
\hline Death & $1(0.1)$ & $3(1.6)$ & .017 & $1(1.2)$ & $0(0.0)$ & 1.000 \\
\hline
\end{tabular}

Abbreviations: ASA, American Anesthesiologists Association; COPD, chronic obstructive pulmonary disease; CVA, cerebral vascular accident; PM, propensity-matched. *PM control cohort was generated by controlling for age, sex, smoking, COPD, dyspnea, hypertension, acute renal failure, disseminated cancer, wound infections, dialysis, ASA.

Bold values indicate statistical significance at $\mathrm{p}<0.05$

dures. ${ }^{15}$ Experimental evidence suggests that glucocorticoids may increase levels of coagulation factors that could contribute to these increased rates of thromboembolic events. ${ }^{16,17}$ In light of the increased rates of $\mathrm{PE}$, it is not unreasonable to have a lower threshold of suspicion for PE and consideration of appropriate chemical prophylaxis in patients on chronic steroid therapy undergoing ACDF.

The present study demonstrated increased rates of septic shock in the steroid cohort compared with the nonsteroid cohort following ACDF for cervical stenosis. Steroid use is known to have negative effects on immunological defense mechanisms, causing secondary immunodeficiency by way of altering immune cell trafficking/functions and decreasing cytokine production. ${ }^{18}$ Steroids bind to glucocorticoid receptors, altering cellular physiology by inhibiting or stimulating gene expression to overall inhibit inflammation. ${ }^{19-21}$ In addition, chronic immunosuppressive therapy can lead to severe lymphopenia and predispose individuals to septic shock. ${ }^{22}$ Prior studies ${ }^{23,24}$ have demonstrated that preoperative steroid use independently increased the risk for infectious complications. Glucocorticoids are widely used to noninvasively treat inflammatory and autoimmune conditions of the spine. ${ }^{21}$ Therefore, with steroid use being commonly implicated in inflammatory spinal conditions, it is important to factor the impacts of prior immunosuppressant therapy in subsequent surgical management.

The rate of these postoperative complications increased by chronic steroid use are most likely contributing to an overall increase in rates of mortality as well. Prior studies ${ }^{25,26}$ have demonstrated increased mortality risk for patients on chronic steroid therapy for various intra-abdominal surgeries. However, there is a lack of literature demonstrating this increased relationship in spinal procedures. In light of the increased rates of mortality, orthopedic surgeons and neurosurgeons should carefully weigh the risks and benefits of potential ACDF to treat cervical stenosis for patients on chronic steroid therapy. Nonoperative treatment options should be thoroughly discussed, and the patient's overall quality of health must be carefully considered. Surgeons may wish to carefully consider the decision to place patients on steroids preoperatively to control radicular and myelopathic symptoms, because the results of our study suggest that there is an increased risk of various postoperative complications. 
There are various limitations to this current retrospective study. Although baseline differences in demographics and preoperative comorbidities were controlled for using propensity-score matching, steroid users may have presented with worse overall health for which the database could not account. Although propensity-score matching is a widely accepted method of matching, the demographics and preoperative comorbidities provided by the ACS NSQIP database do not capture the entire medical history of each of these patients, highlighting a limitation in the granularity of information provided by the database. The increased rates of complications seen in steroid users may be explained by the presence of other medical conditions, such as rheumatoid arthritis and inflammatory bowel disease. Although we attempted to control for baseline differences in demographics and comorbidities to the best of our ability with the information provided by the database, this limitation underscores the need for future research to truly elucidate the impact of chronic steroid usage in outcomes following ACDF for cervical stenosis. In addition, the ACS NSQIP database does not include information as to which specific immunosuppressants were used in each surgical case. Although steroid use is the most common modality of immunosuppression, more targeted immunosuppressants are continuously being developed. It is possible that not differentiating the specific type of immunosuppression used can introduce confounding variables to this study. Furthermore, the ACS NSQIP database does not provide the medical condition for which patients were prescribed immunosuppressive therapy. Finally, the lack of differences seen in complication rates between 1- and 2-level ACDF and 3-level ACDF may be better explained by a significantly smaller sample size in the multilevel fusion cohort. The number of levels fused may serve as a proxy for complexity of the procedure, and one may presume that patients undergoing multilevel fusions would be at a higher risk for complications. However, our results do not support this presumption and must be considered in light of the small sample size within the propensity-matched cohort. Future research with larger cohorts may shed additional insight on the impact of chronic steroid usage on outcomes following ACDF involving 3 or more levels.

\section{CONCLUSION}

It is important to consider the preoperative history of steroid/immunosuppressant use when evaluating candidate patients for ACDF to treat cervical stenosis. Previous literature has reported the association between steroid use in various surgical and spinal procedures and infectious complications. This study demonstrated increased rates for PEs, septic shock, cardiac events, and mortality in patients on chronic steroid therapy. Future research may investigate steroid tapering protocols that reduce the rate or risk of infection and other postoperative complications in the subset of immunosuppressed ACDF patients. The risks and benefits of surgery should be carefully weighed, and nonoperative management options should be thoroughly discussed prior to operative intervention. Presurgical involvement of cardiology, consideration of postoperative DVT chemoprophylaxis, and inpatient monitoring of these patients is recommended. Furthermore, consideration of these data will be crucial in determining bundled payment plans in the United States.

\section{REFERENCES}

1. Mamata H, Jolesz FA, Maier SE. Apparent diffusion coefficient and fractional anisotropy in spinal cord: age and cervical spondylosis-related changes. J Magn Reson Imaging. 2005;22:38-43. https://doi.org/10.1002/jmri.20357

2. Hadjipavlou AG, Tzermiadianos MN, Bogduk N, Zindrick MR. The pathophysiology of disc degeneration: a critical review. J Bone Joint Surg Br. 2008;90:1261-1270. https://doi.org/10.1302/0301-620X.90B10.20910

3. Tetreault LA, Karadimas S, Wilson JR, et al. The natural history of degenerative cervical myelopathy and the rate of hospitalization following spinal cord injury: an updated systematic review. Global Spine J. 2017;S728-S734. https:// doi.org/10.1177/2192568217700396

4. Gibson J, Nouri A, Kreuger B, et al. Degenerative cervical myelopathy: a clinical review. Yale J Biol Med. 2018;91:43-48.

5. Witiw DC, Tetreault LA, Smieliauskas F, et al. Surgery for degenerative cervical myelopathy: a patient-centered quality of life and health economic evaluation. Spine J. 2017;17:15-25. https://doi.org/ 10.1016/j.spinee.2016.10.015

6. Reiter MF, Boden SD. Inflammatory disorders of the cervical spine. Spine (Phila Pa 1976). 1998;23:2755-2766.

7. Kato S, Nouri A, Wu D, et al. Comparison of anterior and posterior surgery for degenerative cervical myelopathy: an MRI-based propensity-score-matched analysis using data from the prospective multicenter AOSpine CSM North America and international studies. J Bone Joint Surg Am. 2017;99:1013-1021. https://doi.org/10.2106/JBJS.16.00882

8. Cunningham J. Pathogenesis and prevention of bone loss in patients who have kidney disease and receive long-term 
immunosuppression. J Am Soc Nephrol. 2007;18:223-234. https://doi.org/10.1681/ASN.2006050427

9. American College of Surgeons National Surgical Quality Improvement Program. User guide for the 2012 ACS NSQIP participant use data file (PUF). October 2013. https://www.facs. org/-/media/files/quality-programs/nsqip/ug12.ashx. Accessed May 1, 2018.

10. White SJW, Carrillo O, Cheung ZB, Ranson WA, Cho SK. The effects of preoperative steroid therapy on perioperative complications after elective anterior lumbar fusion. World Neurosurg. 2019;126:e314-e322.https://doi.org/10.1016/j.wneu. 2019.02.048

11. Liu MM, Reidy AB, Saatee S, Collard CD. Perioperative steroid management-approaches based on current evidence. Anesthesiology. 2017;127:166-172. https://doi.org/10.1097/ ALN.0000000000001659

12. Kelly KN, Domajnko B. Perioperative stress-dose steroids. Clin Colon Rectal Surg. 2013;26:163-167. https://doi. org/10.1055/s-0033-1351132

13. Johannesdottir SA, Horvath-Puho E, Dekkers OM, et al. Use of glucocorticoids and risk of venous thromboembolism: a nationwide population-based case-control study. JAMA Intern Med. 2013;173:743-752. https://doi.org/10.1001/jamaintern med.2013.122

14. Stuijver DJF, Majoor CJ, van Zaane B, et al. Use of oral glucocorticoids and the risk of pulmonary embolism: a population-based case-control study. Chest. 2013;143:13371342. https://doi.org/10.1378/chest.12-1446

15. Lieber BA, Han J, Appelboom G, et al. Association of steroid use with deep venous thrombosis and pulmonary embolism in neurosurgical patients: a national database analysis. World Neurosurg. 2016;89:126-132. https://doi.org/ 10.1016/j.wneu.2016.01.033

16. Squizzato A, Gerdes VE, Ageno W, Büller HR. The coagulation system in endocrine disorders: a narrative review. Intern Emerg Med. 2007;2:76-83. https://doi.org/10.1007/ s11739-007-0026-X

17. van Zaane B, Nur E, Squizzato A, et al. Systematic review on the effect of glucocorticoid use on procoagulant, anticoagulant and fibrinolytic factors. J Thromb Haemost. 2010;8:2483-2493. https://doi.org/10.1111/j.1538-7836.2010. 04034.x

18. Besedovsky L, Linz B, Born J, et al. Mineralocorticoid receptor signaling reduced numbers of circulating human naïve $\mathrm{T}$ cells and increases their CD62L, CCR7, and CXCR4 expression. Eur J Immunol. 2014;44:1759-1769. https://doi. org/10.1002/eji.201344265

19. Oakley RH, Cidlowski JA. The biology of the glucocorticoid receptor: new signaling mechanisms in health and disease. J Allergy Clin Immunol. 2013;132(5):1033-1044. https://doi.org/10.1016/j.jaci.2013.09.007

20. Biola A, Pallardy M. Mode of action of glucocorticoids. Presse Medicale. 2000;29:215-223.

21. Coutinho AE, Chapman KE. The anti-inflammatory and immunosuppressive effects of glucocorticoids, recent developments and mechanistic insights. Mol Cell Endocrinol. 2011;335:2-13. https://doi.org/10.1016/j.mce.2010.04.005

22. Gluck T, Kiefmann B, Grohmann M, Falk W, Straub $\mathrm{RH}$, Scholmerich, J. Immune status and risk for infection in patients receiving chronic immunosuppressive therapy. $J$ Rheumatol. 2005;32:1473-1480.

23. Cloney MB, Garcia RM, Smith ZA, Dahdaleh NS. The effect of steroids on complications, readmission, and reoperation after posterior lumbar fusion. World Neurosurg. 2018;110:e526-e533. https://doi.org/10.1016/j.wneu.2017.11. 030

24. Waljee AK, Rogers MA, Lin P, et al. Short term use of oral corticosteroids and related harms among adults in the United States: population-based cohort study. BMJ. 2017;357:j1415. https://doi.org/10.1136/bmj.j1415

25. Moghadamyeghaneh Z, Hanna MH, Blondet JJ, et al. Impact of chronic steroid use on outcomes of colorectal surgery. Am J Surg. 2015;210:1003-1009. https://doi.org/10. 1016/j.amjsurg.2015.07.002

26. Kaplan JA, Schecter SC, Rogers SJ, Lin MYC, Posselt AM, Carter JT. Expanded indications for bariatric surgery: should patients on chronic steroids be offered bariatric procedures? Surg Obes Relat Dis. 2017;13:35-40. https://doi. org/10.1016/j.soard.2015.10.086

Disclosures and COI: The authors declare no conflict of interest and have not received any funding for this work.

Corresponding Author: Ryan Lee, MBA, The George Washington University School of Medicine and Health Sciences, 2300 I Street NW, Washington, DC 20052. Phone: (201) 921-0023; Email: ryanlee@gwu.edu.

Published 11 November 2020

This manuscript is generously published free of charge by ISASS, the International Society for the Advancement of Spine Surgery. Copyright (C) 2020 ISASS. To see more or order reprints or permissions, see http://ijssurgery.com. 